\title{
Static Color-Coulomb Force
}

\author{
Attilio Cucchieri* and Daniel Zwanziger ${ }^{\dagger \dagger}$ \\ Physics Department \\ New York University \\ New York, NY 10003
}

(March 21, 2018)

\begin{abstract}
The static color-Coulomb interaction potential is calculated as the solution of a non-linear integral equation which arises in the Hamiltonian Coulomb gauge when the restriction to the interior of the Gribov horizon is implemented. The potential obtained is in qualitative agreement with expectations, being Coulombic with logarithmic corrections at short range, and confining at long range. The values obtained for the string tension and $\Lambda_{\overline{M S}}$ are in semi-quantitative agreement with lattice Monte Carlo and phenomenological determinations.
\end{abstract}

Pacs numbers: 12.38.Gc, 12.39.Pn

The interaction energy $E(r)$ of a pair of heavy quarks at separation $r$ is a prominent feature of QCD phenomenology. From fits to the charmonium and bottonium spectra [1,2], $E(r)$ is well-known in the range $1(\mathrm{GeV})^{-1}<r<4(\mathrm{GeV})^{-1}$. To calculate $E(r)$ from first principles, one makes a Born-Oppenheimer approximation, in which heavy quarks move slowly, while gluons and light quarks rapidly adjust to the instantaneous position of the heavy quarks. In this approximation, $E(r)$ is the potential energy that appears in a non-relativistic Schroedinger equation that describes the slow motion of the heavy quarks, and from which the phenomenological fit for $E(r)$ is obtained. It is calculated as the gauge-invariant ground-state energy $\mathcal{H} \Psi_{0}=E(r) \Psi_{0}$, where $\mathcal{H}$ is the field-theoretic QCD Hamiltonian that describes the dynamics of gluons and light quarks in the presence of an external quark-anti-quark pair at fixed separation $r$. In the absence of light or dynamical quarks, it is generally believed that $E(r)$ grows linearly at large $r$, $E(r) \sim K r$, with string-tension $K$, and this belief is supported by lattice-gauge calculations [3]. Pair production of dynamical quarks from the vacuum causes "breaking" of the string at large $r$. However, in the above energy range where $E(r)$ is known phenomenologically, string-breaking is not yet manifest, and we shall ignore dynamical quarks in the following.

The Coulomb-gauge is a so-called "physical" gauge in which the conjugate dynamical variables are the 3dimensionally transverse fields $A=A^{t r}$ and $\left(-E^{t r}\right)$, where $E_{x, i}^{a}=\left(E^{t r}\right)_{x, i}^{a}-\left(\nabla_{i} \varphi^{a}\right)_{x}$ and $\varphi$ is the color-Coulomb operator. Although the Coulomb-gauge is not known to be renormalizable, a lattice Coulomb-gauge Hamiltonian has been derived recently from the transfer matrix of Wilson's gauge-invariant Euclidean lattice gauge theory [4], so that gauge-invariant quantities such as $E(r)$ have the same continuum limit as in Wilson's theory. For brevity, equations will be written formally in continuum notation. The exact lattice equations may be found in $\llbracket$ ].

The transversality condition on $A$ is not a complete gauge fixing because of the existence of Gribov copies [5] To obtain a complete gauge fixing, we adopt the minimal Coulomb gauge. In this gauge, the set $\Lambda$ of gauge-fixed configurations, called the fundamental modular region (FMR), consists of configurations $A$ for which the Hilbert norm $\|A\|^{2} \equiv \int d^{3} x|A|^{2}$ of $A$ is minimized with respect to local gauge transformations $g: \Lambda \equiv\left\{A:\|A\| \leq\left\|A^{g}\right\|\right.$ for all $\left.g\right\}$. Here $A^{g}=g^{\dagger} A g+g^{\dagger} \nabla g$ is the gauge transform of $A$. Configurations with this property are transverse, $A=A^{t r}-$ so this gauge falls into the class of Coulomb-gauges - and moreover the Faddeev-Popov (FP) operator $M(A) \equiv-D \cdot \nabla$ is symmetric $M(A)=M^{\dagger}(A)$ and positive $M(A) \geq 0$ for all $A \in \Lambda$ 田. Here $D=D(A)$ is the gauge-covariant derivative $D^{a, c}(A) \equiv \delta^{a, c} \nabla+g_{0} f^{a b c} A^{b}$. In the following we write $A$ for $A^{t r}$.

With $E=E^{t r}-\nabla \phi$, the non-Abelian Gauss's law constraint, $D \cdot E=g_{0} \rho_{q u}$, may be written $M \phi=g_{0} \rho$. Here $\rho_{q u}$ is the color-charge density of the quarks, and $\rho^{a} \equiv-f^{a b c} A^{t r, b} E^{t r, c}+\rho_{q u}^{a}$ is the color-charge density of the dynamical degrees of freedom. Because $M(A)$ is a positive operator in the minimal Coulomb gauge, the color-Coulomb field $\phi$ may be expressed unambiguously in terms of the dynamical degrees of freedom, $\phi=g_{0} M^{-1} \rho$, and so also the color-electric field $E=E^{t r}-\nabla \phi$. For wave functionals $\Phi(A)$ and $\Psi(A)$ defined for $A$ in $\Lambda$, and with $E^{t r}=i \delta / \delta A^{t r}$, the Coulomb-gauge Hamiltonian [6] is defined by the quadratic form

$$
\left(\Phi, H_{\mathrm{coul}} \Psi\right)=\int_{\Lambda} d A^{t r} \sigma \int d^{3} x 2^{-1}\left[\left(E_{i}^{a} \Phi\right)^{*} E_{i}^{a} \Psi+\Phi^{*} B_{i}^{a} B_{i}^{a} \Psi\right],
$$

\footnotetext{
*Electronic address: attilioc@asterix.physics.nyu.edu

${ }^{\dagger}$ Electronic address: zwanzige@acf2.nyu.edu

${ }^{\ddagger}$ Research supported in part by the National Science Foundation under grant no. PHY93-18781
} 
where $E$ has just been defined, $B_{i}^{a} \equiv \nabla_{j} A_{k}-\nabla_{k} A_{j}+f^{a b c} A_{j}^{b} A_{k}^{c}$ for $i, j$, and $k$ cyclic, $\sigma \equiv \operatorname{det}[M(A) / M(0)]$, and the quark Hamiltonian is suppressed. This Hamiltonian is symmetric with respect to the inner product $(\Phi, \Psi)=$ $\int_{\Lambda} d A^{t r} \sigma \Phi^{*} \Psi$, and $\sigma(A)$ is positive because $M(A)$ is.

The main novel ingredient in the present approach is the implementation of the restriction of the preceding integrals to $\Lambda$, a non-perturbative effect [7]. For finite quantization-volume $V$, the exact boundary of $\Lambda$ is not known. However, in [ [4] it is argued that, for periodic boundary conditions and in the limit of large $V$, this region is adequately approximated by $G / V \leq 0$, and moreover that this restriction may be implemented by use of the effective Hamiltonian $H_{\text {coul }} \Rightarrow H_{\text {eff }}=H_{\text {coul }}+\gamma_{0} G$. Here the horizon function $G(A)$ is defined for structure group $S U(N)$ by

$$
G(A) \equiv \int d^{3} x d^{3} y D_{x, i}^{a, c} D_{y, i}^{a, d}\left(M^{-1}\right)^{c, d}(x, y)-3\left(N^{2}-1\right) V .
$$

The term $\gamma_{0} G$ makes the wave-functionals in the Fock space of $H_{\text {eff }}$ vanish rapidly outside $\Lambda$, so the restriction on the integrals may be ignored, $\int_{\Lambda} d A^{t r} \Rightarrow \int d A^{t r}$. Here $\gamma_{0}$ is a thermodynamic parameter that sets the scale for hadronic masses. Its value is determined by the horizon condition $\langle G\rangle / V=0$. Here and below, the expectation-value is taken in the ground state of $H_{\text {eff }}$. The horizon condition expresses the fact that, for large $V$, the probability gets concentrated on the boundary $G / V=0$.

The Coulomb energy

$$
E_{\text {coul }}=\int d A^{t r} \sigma \int d^{3} x 2^{-1}\left|g_{0} \nabla\left(M^{-1} \rho\right) \Phi\right|^{2}
$$

contributes additively to the energy of a state $\Phi$, the remaining terms being positive. Consider the contribution to $E_{\text {coul }}$ from the part of $M^{-1}$ which is diagonal in momentum space,

$$
V^{-1}\left(M^{-1}\right)_{q, a: q, b}=\int d^{3} y e^{i q \cdot y}\left[V^{-1} \int d^{3} x\left(M^{-1}\right)_{x, a ; x+y, b}\right]
$$

For each $y$, the term in brackets is a bulk quantity per unit volume. By translation invariance, its covariance matrix is of order $V^{-1}$. Consequently, for large $V$, it approaches its mean-field value

$$
V^{-1}\left(M^{-1}\right)_{q, a ; q, b}=C(q) \delta^{a, b}+\mathcal{O}\left(V^{-1 / 2}\right),
$$

where

$$
C(q) \delta^{a, b}=\int d^{3} y e^{i q \cdot y}\left\langle\left(M^{-1}\right)_{x, a ; x+y, b}\right\rangle
$$

is the 3 -dimensional FP propagator. Thus $\phi$ and $E_{\text {coul }}$ receive the contributions $\tilde{\phi}(q)=g_{0} C(q) \tilde{\rho}(q)$ and

$$
\int d A^{t r} \sigma(2 V)^{-1} \sum_{q} q^{2}\left|g_{0} C(q) \tilde{\rho}(q) \Phi\right|^{2}
$$

where the color-charge density operator $\tilde{\rho}(q)$ satisfies $\lim _{q \rightarrow 0} \tilde{\rho}^{a}(q)=Q^{a}$. Here $Q^{a}$, with $\left[Q^{a}, Q^{b}\right]=f^{a b c} Q^{c}$, is the total color-charge.

The FP propagator is of the form $C^{-1}(q)=q^{2}-q_{i} \Sigma_{i, j} q_{j}$, where $\Sigma_{i, j}(q)$ is a self energy. The horizon condition is equivalent 啗 to the condition $\Sigma_{i, j}(0)=\delta_{i, j}$. This gives $C^{-1}(q)=q_{i}\left[\Sigma_{i, j}(0)-\Sigma_{i, j}(q)\right] q_{j}$, so $C(q)$ is more singular at $q=0$ than $q^{-2}$. We conclude that the restriction to the FMR produces a long-range color-Coulomb field, as originally foreseen by Gribov [5].

The self-energy $\Sigma$ satisfies a Schwinger-Dyson equation which we write symbolically $q \Sigma q=R(q) \equiv g_{0}^{2} \int \Gamma_{0} D C \Gamma$, which gives $C^{-1}(q)=R(0)-R(q)$. Here $D$ is the gluon propagator, and $\Gamma$ and $\Gamma_{0}$ are the exact and zeroth-order ghost-ghost-gluon vertex functions. When $R$ is evaluated in the ground state of $H_{\text {eff }}$, this equation [8] provides the mean-field self-consistency condition (MSC) that determines the mean-field function $C(q)$.

So far our results are exact, but in the following we resort to approximations to solve the MSC. This equation contradicts the usual perturbative expansion, as one sees from the appearance of $\left(g_{0}\right)^{2}$ in $R$, whereas $C, D$, and $\Gamma$ are nominally of leading order $\left(g_{0}\right)^{0}$. To obtain an expansion in powers of $g_{0}$ that is consistent with the MSC, we assume that $C(q)$ is of leading order $\left(g_{0}\right)^{-1}$, namely $C(q)=g_{0}^{-1} u(q)+O(1)$, and that all other correlation functions are analytic in $g_{0}$. To leading order, the MSC is an integral equation that determines $u(q)$ :

$$
u^{-1}(q)=N(2 \pi)^{-3} \int d^{3} k D_{0}(k)\left[q^{2}-(q \cdot k)^{2} / k^{2}\right][u(k)-u(k-q)] .
$$


The kernel $D_{0}$ is the gluon propagator

$$
D_{0}(k)\left(\delta_{i, j}-k_{i} k_{j} / k^{2}\right) \delta^{b, c}=\int d^{3} x e^{-i k \cdot x}\left\langle A_{x+y, i}^{b} A_{y, j}^{c}\right\rangle_{0},
$$

evaluated in the ground state of

$$
H_{0} \equiv \sum_{k, \lambda} \omega_{k} a_{k, \lambda}^{\dagger} a_{k, \lambda}+(2 V)^{-1} \sum_{k} \tilde{\rho}_{-k}^{a} v_{k} \tilde{\rho}_{k}^{a}
$$

where $a_{k, \lambda}^{\dagger}$ and $a_{k, \lambda}$ are creation and annihilation operators for $A^{t r}$ and $E^{t r}, \lambda$ is a two-valued polarization index, and $\omega_{k}=\left(k^{2}+\mu^{4} u_{k}\right)^{1 / 2}$. This is the zeroth-order part of $H_{\text {eff }}$, obtained by systematically expanding $H_{\text {eff }}$ in powers of $g_{0}$, with $\gamma_{0}$ scaled according to $\gamma_{0}=\left(2 N g_{0}\right)^{-1} \mu^{4}$. In addition to a harmonic oscillator Hamiltonian, $H_{0}$ also contains a color-Coulomb interaction Hamiltonian with interaction potential $v_{k} \equiv k^{2} u_{k}^{2}$ that is independent of $g_{0}$, as is consistent with dimensional transmutation.

This term prevents us from calculating $D_{0}(k)$ exactly. We neglect it, so $D_{0}$ is approximated by $D_{0}^{(0)}(k) \equiv\left(2 \omega_{k}\right)^{-1}$, and we call $w_{q} \equiv u_{q}^{(0)}$ the solution to (8) with kernel $D_{0}^{(0)}$. We expect that $D_{0}(k)$ is even more suppressed at low $k$ than $D_{0}^{(0)}(k)$, so by (8) $u_{q}$ is enhanced at low $q$ compared to $w_{q}$ corresponding to an even longer range force than we find.

The asymptotic form of $w_{q}$ at high and low $q$ has been determined analytically [4], and it has been found numerically for intermediate $q$ with $2 \%$ accuracy (see [9, 10 for details). We write $w(q)=g_{c}(q) / q^{2}$, where $g_{c}(q)$ is a renormalized running Coulomb-coupling constant, and we express our results in terms of the static color-Coulomb potential $v_{q}=$ $q^{2} w^{2}(q)=g_{c}^{2}(q) / q^{2}$, which appears in $H_{0}$, and the Fourier transform of $v_{q}$ (normalized for a pair of external quarks), which is given (apart from an additive constant) by

$$
V(r)=-\left(N^{2}-1\right)\left(4 N \pi^{2}\right)^{-1} \int_{0}^{\infty} d q g_{c}^{2}(q)(q r)^{-1} \sin (q r),
$$

corresponding to a force $f(r)=-V^{\prime}(r)$.

The asymptotic form of $g_{c}$ at high $q$ is given by

$$
g_{c}^{-2}=b_{c} t+6^{-1} b_{c} \ln \left(b_{c} t\right)+\mathcal{O}\left(t^{-1} \ln t\right),
$$

where $t=\ln (q / m), b_{c}=\left(3 \pi^{2}\right)^{-1} N, m=C \mu$, and $C$ is a constant. The first and second terms are of the form of the one- and two-loop contributions to the running coupling constant $g_{\mathrm{rg}}$ of the perturbative renormalization group, but the coefficients are different. This difference arises because at high momentum the interaction is not purely static Coulombic, and it is verified in [4] that the difference in the first coefficient is correctly accounted for by terms that are neglected here. The coefficient of the second term is a new result. The limiting behavior of the force $f(r)$ at small $r$ is given by

$$
f(r) \approx 3 \pi\left(N^{2}-1\right)\left(4 N^{2} r^{2}\right)^{-1}\left[\log \left(\Lambda_{R}^{2} r^{2}\right)+3^{-1} \log \log \left(\lambda^{2} r^{2}\right)\right]^{-1}
$$

where $\Lambda_{R} \equiv e^{\gamma-1} m, \gamma$ is Euler's constant, and $\lambda$ is a constant.

The confinement properties of the theory in the present approximation are determined by the asymptotic form of $g_{c}(q)$ at low $q$, which is given by $g_{c}(q)=B(\mu / q)^{4 / 3}$, where $B^{-3 / 2}=N \pi^{-2} \Gamma(8 / 3) \Gamma(2 / 3) / \Gamma(16 / 3)$ (see [4]). This corresponds to a color-Coulomb potential $V(r) \sim r^{5 / 3}$ which rises more rapidly with $r$ than a string tension $K r$. This somewhat surprising result may be an artifact of the approximations made. On the other hand $V(r)$, which appears in the (approximate) quantum-field theoretic Coulomb-gauge Hamiltonian, must be distinguished from the gauge-independent quark-pair energy $E(r)$ discussed in the introduction, and it is shown in [4] that $E(r) \leq V(r)$. If $V(r)$ does grow more rapidly than $K r$ at large $r$, then the wave-function $\Psi\left(A^{t r}\right)$, whose defining property is to minimize $E(r)$, adjusts itself so $E(r)$ rises no more rapidly than $K r$ [1]. It does so by changing the two superposed spherically symmetric long range color-Coulomb fields into a flux tube.

We shall compare our results for $V(r)$ with phenomenological fits to $E(r)$, to see if there is a range of "small" $r$ for which $V(r)$ agrees with $E(r)$, as suggested by asymptotic freedom. To this end, we consider two phenomenological models: the Cornell potential [1] and the Richardson potential [2], both of which fit the $c \bar{c}$ and $b \bar{b}$ spectra well.

To connect dimensionless quantities and the real world, we fix the length scale by using Sommer's [12] dimensionless phenomenological relation $r_{0}^{2} f\left(r_{0}\right)=-1.65$ which holds for the Cornell force at $r_{0} a \equiv R_{0}=2.48(\mathrm{GeV})^{-1}$. From the value of $a$ in $(\mathrm{GeV})^{-1}$ we obtain the string tension $\sqrt{\sigma} \equiv \sqrt{\min _{r}[-f(r)]} a^{-1}=518 \mathrm{MeV}$. [By this definition, 
we are evaluating $\sigma$ where $f^{\prime}(r)=0$, i.e where the potential is approximately linear.] It is not easy to estimate an uncertainty for the string tension. However, its value seems to depend very weakly on the values of the parameters of our trial solution [9. If we identify the parameter $\Lambda_{R} \equiv e^{\gamma-1} m$ in (13) with the corresponding physical parameter [13], then from the relation [13] $\Lambda_{\overline{M S}}=\Lambda_{R} \exp (-\gamma+1-31 / 66)$ we obtain $\Lambda_{\overline{M S}}=124 \pm 12 \mathrm{MeV}$ (see [9],14]). In Figure 11a we plot our result for $f(r)$, the two Cornell forces [1], and the Richardson force [2]. Our force gets its maximum value at a separation of about $2.5(\mathrm{GeV})^{-1}$ and it is almost constant up to $4(\mathrm{GeV})^{-1}$, the variation being of order $12 \%$.

These results depend on the phenomenological conditions that we have used to set the length scale. If we use the dimensionless relation $r_{0}^{2} f\left(r_{0}\right)=-1.35$, which holds for the Richardson force at $R_{0}=2.48(\mathrm{GeV})^{-1}$, we obtain $\sqrt{\sigma}=468 \mathrm{MeV}, \Lambda_{\overline{M S}}=118 \pm 12 \mathrm{MeV}$ and the plot shown in Figure $1 \mathrm{~b}$. In this case the agreement is even better: our force reaches its maximum value at a separation of about $2.75(\mathrm{GeV})^{-1}$, and its variation at $4(\mathrm{GeV})^{-1}$ is of order $8 \%$.

As an exact result, we have found that the restriction to the fundamental modular region causes an infrared singularity of the color-Coulomb propagator and thus a long-range color-Coulomb potential. Although our calculation of this potential required possibly severe approximations that are describe above, nevertheless it gives results which are in qualitative agreement with phenomenologically determined potentials. The fit is surprisingly good. After setting the scale by Sommer's method, the force is in qualitative agreement with phenomenological models, and the values obtained for the string tension and $\Lambda_{\overline{M S}}$ are in semi-quantitative agreement with lattice Monte Carlo and phenomenological determinations (see [1],3] and [15]). It would appear that the approximate equality $V(r) \approx E(r)$ extends to the range $r<4(\mathrm{GeV})^{-1}$, and moreover that the approximations made in our calculation of $V(r)$ do not qualitatively destroy this agreement. Although there is no a priori reason to expect that vacuum polarization of gluons should not be important in this range, this may not be so surprising after all. For although vacuum polarization (pair production) of quarks does "break" the string, this is not yet manifest for $r<4(\mathrm{GeV})^{-1}$.

We are grateful to Tony Duncan, Martin Schaden and Alberto Sirlin for informative discussions.

[1] E. Eichten et al., Phys. Rev. Lett. 34 (1975) 369; E. Eichten et al., Phys. Rev. D21 (1980) 203; E. Eichten and F. Feinberg, Phys. Rev. D23 (1981) 2724.

[2] J. L. Richardson, Phys. Lett. B82 (1979) 272.

[3] G. S. Bali and K. Schilling, Nucl. Phys. B (Proc. Suppl.) 34 (1994) 147.

[4] D. Zwanziger, Nucl.Phys. B485 (1997) 185.

[5] V. N. Gribov, Nucl. Phys. B139 (1978) 1.

[6] N. Christ and T. D. Lee, Phys. Rev. D22 (1980) 939.

[7] An alternative approach which has been advocated recently is to integrate over all $A^{t r}$ without restriction, but with $\sigma(A)$ as a signed measure [see R. Friedberg et al., Ann. of Phys. 246 (1996) 381].

[8] In the Landau gauge, where $\tilde{Z}_{1}^{\prime}=1$, this equation is invariant under renormalization — the Z's cancel! — and a corresponding property should also hold in the Coulomb-gauge.

[9] A. Cucchieri, Numerical Results in Minimal Lattice Coulomb and Landau Gauges: Color-Coulomb Potential and Gluon and Ghost Propagators, PhD thesis, New York University (May 1996).

[10] A. Cucchieri and D. Zwanziger, Nucl.Phys. B (Proc. Suppl.) 53 (1997) 815.

[11] E. Seiler, Phys. Rev. D22 (1980) 2412.

[12] R. Sommer, Nucl. Phys. B411 (1994) 839.

[13] A. Billoire, Phys. Lett. B104 (1981) 472.

[14] The uncertainty on $\Lambda_{\overline{M S}}$ comes from the uncertainties on the values of $a$, and of the parameter $m$ entering into the definition of $\Lambda_{R}$. By solving equation (8) numerically, we have found the value of $m$ with an estimated accuracy of about $10 \%$. We have considered negligible the uncertainty on $a$.

[15] Particle Data Group, Review of Particle Properties, Phys. Rev. D50 (1994) 1173. 
Forces (case 1)

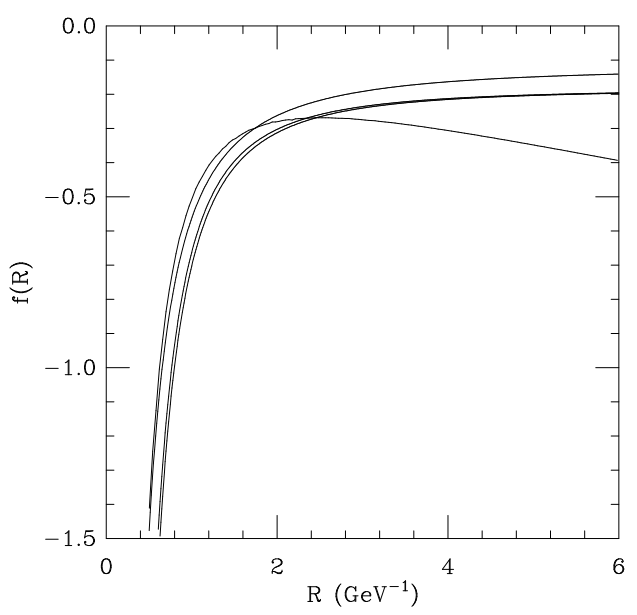

Forces (case 2)

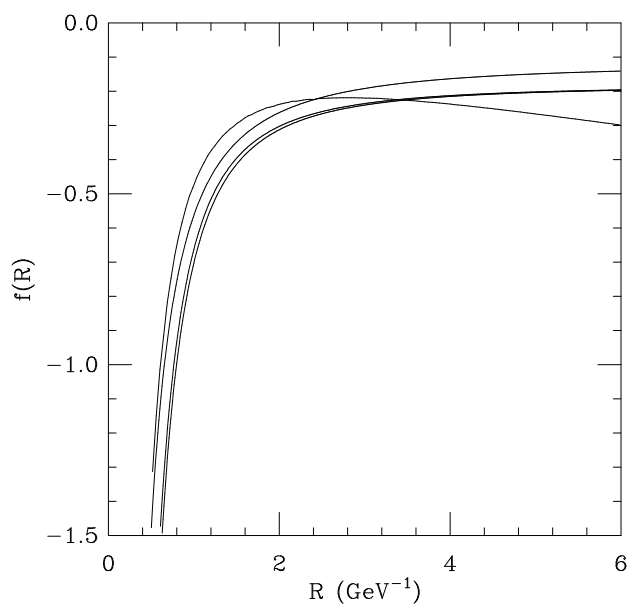

FIG. 1. Plot of: (i) our force $f(R)$ (the curve which is increasing negative at large $R$ ), (ii) the forces (for the $c \bar{c}$ and the $b \bar{b}$ cases) derived from the Cornell potential (the two curves very close to each other), and (iii) the Richardson force (the highest curve). In case 1 we set the length scale by $r_{0}^{2} f\left(r_{0}\right)=-1.65$ from the Cornell force, while in case 2 we use the condition $r_{0}^{2} f\left(r_{0}\right)=-1.35$ from the Richardson force, both at $R_{0}=2.48(\mathrm{GeV})^{-1}$. 